\title{
NEW UPPER BOUNDS FOR RAMANUJAN PRIMES
}

\author{
Anitha Srinivasan and Pablo Ares-Gastesi \\ Saint Louis University and Universidad CEU San Pablo, Spain
}

\begin{abstract}
For $n \geq 1$, the $n^{\text {th }}$ Ramanujan prime is defined as the smallest positive integer $R_{n}$ such that for all $x \geq R_{n}$, the interval $\left(\frac{x}{2}, x\right]$ has at least $n$ primes. We show that for every $\epsilon>0$, there is a positive integer $N$ such that if $\alpha=2 n\left(1+\frac{\log 2+\epsilon}{\log n+j(n)}\right)$, then $R_{n}<p_{[\alpha]}$ for all $n>N$, where $p_{i}$ is the $i^{\text {th }}$ prime and $j(n)>0$ is any function that satisfies $j(n) \rightarrow \infty$ and $n j^{\prime}(n) \rightarrow 0$.
\end{abstract}

\section{INTRODUCTION}

For $n \geq 1$, the $n^{\text {th }}$ Ramanujan prime is defined as the smallest positive integer $R_{n}$, such that for all $x \geq R_{n}$, the interval $\left(\frac{x}{2}, x\right]$ has at least $n$ primes. Note that by the minimality condition, $R_{n}$ is prime and the interval $\left(\frac{R_{n}}{2}, R_{n}\right]$ contains exactly $n$ primes. Let $R_{n}=p_{s}$, where $p_{i}$ denotes the $i^{\text {th }}$ prime. Sondow ([7]) showed that $p_{2 n}<R_{n}<p_{4 n}$ for all $n$, and conjectured that $R_{n}<$ $p_{3 n}$ for all $n$. This conjecture was proved by Laishram ([4]), and the upper bound $p_{3 n}$ improved by various authors ([1], [8]). Subsequently, Srinivasan ([9]) and Axler ([1]) improved these bounds by showing that for every $\epsilon>0$, there exists an integer $N$ such that

$$
R_{n}<p_{[2 n(1+\epsilon)]} \text { for all } n>N .
$$

Using the method in [9] (outlined below), a further improvement was presented by Srinivasan and Nicholson, who proved that

$$
s<2 n\left(1+\frac{3}{\log n+\log (\log n)-4}\right)
$$

2010 Mathematics Subject Classification. Ramanujan primes, upper bounds. Key words and phrases. 11A41, 11N05. 
for all $n>241$. The above result follows from a special case of our main theorem given below. Yang and Togbé ([11]), also used the method in [9], to give tight upper and lower bounds for $R_{n}$ for large $n$ (greater than $10^{300}$ ). For some interesting generalizations of Ramanujan primes the reader may refer to [2], [5] and [6].

The main idea in [9] is to define a function $F(x)$ that is decreasing for $x \geq 2 n$ and that satisfies $F(s)>0$. Then, an $\alpha>2 n$ is found such that $F(\alpha)<0$ for $n>N$, which would imply that $s<\alpha$ for $n>N$ given the decreasing nature of $F$. We employ a variation of this method, where we first show that $F(\alpha)$ is a decreasing function for $n>N$. Then we find an integer greater than $N$ for which $F(\alpha)<0$, which leads us to the desired result. Our main result is the following.

TheOREM 1.1. Let $R_{n}=p_{s}$ and $\epsilon>0$. Let $j(n)>0$ be a function such that $j(n) \rightarrow \infty$ and $n j^{\prime}(n) \rightarrow 0$ as $n \rightarrow \infty$ and let

$$
g(n)=\frac{\log n+j(n)}{\log 2+\epsilon} .
$$

Then there exists a positive integer $N$ such that for all $n>N$, we have $s<\alpha$, where $\alpha=2 n\left(1+\frac{1}{g(n)}\right)$.

Let $\log _{2} x$ denote $\log \log x$. In the following corollary we record a bound obtained with $\epsilon=0.5$, where $j(n)$ is chosen so as to minimize the number of calculations. Similar results can be given for smaller values of $\epsilon$ (with different $j(n)$ ) where the determination of $N$ depends solely on computational power.

COROLlary 1.2. Let $R_{n}=p_{s}$. Then for $n>43$ we have

$$
s<2 n\left(1+\frac{1}{g(n)}\right)
$$

where

$$
g(n)=\frac{\log n+\log _{2} n-\log 2-0.5}{\log 2+0.5}
$$

\section{The BASIC FUnCTIONS AND LEMMAS}

We will use the following bounds for the $k^{\text {th }}$ prime given by Dusart.

LEMMA 2.1. The following hold for the $k^{\text {th }}$ prime $p_{k}$.

1. $p_{k}>k\left(\log k+\log _{2} k-1+\frac{\log _{2} k-2.1}{\log k}\right)$ for all $k \geq 3$.

2. $p_{k}<k\left(\log k+\log _{2} k-1+\frac{\log _{2} k-2}{\log k}\right)$ for all $k \geq 688383$.

Proof. See [3]. 
Let

$$
U(k)=k\left(\log k+\log _{2} k-1+\frac{\log _{2} k-2}{\log k}\right)
$$

and

$$
L(k)=k\left(\log k+\log _{2} k-1+\frac{\log _{2} k-2.1}{\log k}\right) .
$$

Note that $U(x)=L(x)+f(x)$ where $f(x)=\frac{0.1 x}{\log x}$. We define

$$
F(x, n)=U(x)-2 L(x-n)=U(x)-2 U(x-n)+2 f(x-n)
$$

and

$$
G(n)=F(\alpha, n),
$$

where $\alpha=2 n\left(1+\frac{1}{g(n)}\right)$ and $g(n)$ is a function that satisfies $g(n) \geq 1$ and $g(n) \rightarrow \infty$ as $n \rightarrow \infty$.

LEMma 2.2. Let $R_{n}=p_{s}$. Then the following hold.

1. $p_{s-n}<\frac{1}{2} p_{s}$.

2. $2 n<s<2.4 n$ for all $n>43$.

3. $F(x, n)$ is a decreasing function for all $x \geq 2 n$ and $F(s, n)>0$ for $n \geq 688383$.

Proof. For parts 1 and 2 see [9, Lemma 2.1] and [9, Remark 2.1] respectively. For part 3 see [11].

The following lemma contains useful results that include an expression for the derivative $G^{\prime}(n)$ in terms of the function $U(x)$.

Lemma 2.3. Let $A=U^{\prime}(\alpha)-U^{\prime}(\alpha-n)$. Then the following hold.

1. $A=A(n) \rightarrow \log 2$ as $n \rightarrow \infty$.

2. $\frac{1}{2} G^{\prime}(n)=A+f^{\prime}(\alpha-n)+\left(\frac{n}{g(n)}\right)^{\prime}\left(A-U^{\prime}(\alpha-n)+2 f^{\prime}(\alpha-n)\right)$.

3. $L^{\prime}(x)>\log x+\log _{2} x$ for $x>20$.

4. $A+f^{\prime}(\alpha-n)-\log 2<\log \left(\frac{\log \alpha}{\log (\alpha-n)}\right)+\frac{\log _{2} \alpha}{\log \alpha}+\frac{1.1}{\log (\alpha-n)}+\frac{\log _{2}(\alpha-n)}{\log ^{2}(\alpha-n)}$.

Proof. We have

$$
U^{\prime}(x)=\log x+\log _{2} x-\frac{1}{\log x}+\frac{3}{\log ^{2} x}-\frac{\log _{2} x}{\log ^{2} x}+\frac{\log _{2} x}{\log x}
$$

and hence

$$
A=\log \left(\frac{\alpha}{\alpha-n}\right)+\log \left(\frac{\log (\alpha)}{\log (\alpha-n)}\right)+t(n),
$$

where $t(n) \rightarrow 0$ as $n \rightarrow \infty$. As $\alpha=2 n\left(1+\frac{1}{g(n)}\right)$ and $g(n) \rightarrow \infty$, we have $A \rightarrow \log 2$. 
For the second part of the lemma, $G(n)=U(\alpha)-2 U(\alpha-n)+2 f(\alpha-n)$, which gives $G^{\prime}(n)=U^{\prime}(\alpha) \alpha^{\prime}-2 U^{\prime}(\alpha-n)\left(\alpha^{\prime}-1\right)+2 f^{\prime}(\alpha-n)\left(\alpha^{\prime}-1\right)$. As $\alpha^{\prime}=2+2\left(\frac{n}{g(n)}\right)^{\prime}$, we have

$$
\frac{1}{2} G^{\prime}(n)=U^{\prime}(\alpha)\left(1+\left(\frac{n}{g}\right)^{\prime}\right)+\left(1+2\left(\frac{n}{g}\right)^{\prime}\right)\left(f^{\prime}(\alpha-n)-U^{\prime}(\alpha-n)\right)
$$

and the result follows by the definition of $A$.

For part 3 we have

$$
L^{\prime}(x)=\log x+\log _{2} x+\frac{\log _{2} x}{\log x}-\frac{\log _{2} x}{\log ^{2} x}-\frac{1.1}{\log x}+\frac{3.1}{\log ^{2} x}
$$

from which the claim follows as for $n>20$ we have $\frac{\log _{2} x}{\log x}-\frac{\log _{2} x}{\log ^{2} x}-\frac{1.1}{\log x}>0$.

For the last part, we have

$$
\begin{aligned}
& A-\log 2+f^{\prime}(\alpha-n) \\
& \quad=\log \left(\frac{\log \alpha}{\log (\alpha-n)}\right)+\frac{\log _{2} \alpha}{\log \alpha}+\frac{1.1}{\log (\alpha-n)}+\frac{\log _{2}(\alpha-n)}{\log ^{2}(\alpha-n)}+T,
\end{aligned}
$$

where

$$
\begin{aligned}
T= & \log \left(\frac{1+\frac{1}{g(n)}}{1+\frac{2}{g(n)}}\right)-\frac{\log _{2}(\alpha-n)}{\log (\alpha-n)}-\frac{1}{\log \alpha} \\
& -\frac{\log _{2} \alpha}{\log ^{2} \alpha}+\frac{3}{\log ^{2} \alpha}-\frac{3.1}{\log ^{2}(\alpha-n)}<0
\end{aligned}
$$

as $\frac{3}{\log ^{2} \alpha}-\frac{3.1}{\log ^{2}(\alpha-n)}<0$.

\section{Proofs OF MAIN RESUlts}

The following lemma shows that $G^{\prime}(n)$ is a decreasing function for large $n$, which is crucial in the proof of Theorem 1.1.

LEMMA 3.1. Let $\epsilon>0$ and

$$
g(n)=\frac{\log n+j(n)}{\log 2+\epsilon}
$$

where $j(n)>0$ is a function that satisfies $j(n) \rightarrow \infty$ and $n j^{\prime}(n) \rightarrow 0$ as $n \rightarrow \infty$. Then $G^{\prime}(n) \rightarrow-2 \epsilon$.

Proof. We have

$$
\left(\frac{n}{g(n)}\right)^{\prime}=\frac{(\log 2+\epsilon)\left(\log n+j(n)-1-n j^{\prime}(n)\right)}{(\log n+j(n))^{2}}
$$

and therefore $\left(\frac{n}{g(n)}\right)^{\prime} \rightarrow 0$ as $n \rightarrow \infty$. By our assumption on $j(n)$ it follows (using L'Hôpital's rule) that $\frac{j(n)}{\log n} \rightarrow 0$ which gives $\left(\frac{n}{g(n)}\right)^{\prime} \log (\alpha-n) \rightarrow$ 
$\log 2+\epsilon\left(\right.$ as $\left.\frac{\log (\alpha-n)}{\log n} \rightarrow 1\right)$. It is easy to see that $\left(\frac{n}{g(n)}\right)^{\prime} \log _{2}(\alpha-n) \rightarrow 0$. It follows that $\left(\frac{n}{g(n)}\right)^{\prime} U^{\prime}(\alpha-n) \rightarrow \log 2+\epsilon$ (see equation (2.1)). Lastly note that $f^{\prime}(x) \rightarrow 0$ as $x \rightarrow \infty$. The result follows now on using all the above and the fact that $A \rightarrow \log 2$ (Lemma 2.3 part 1) in part 2 of Lemma 2.3.

Proof of TheOREM 1.1. We will first show that there exists a positive integer $N$, such that $G(n)<0$ for $n>N$. We have $G^{\prime}(n) \rightarrow-2 \epsilon$ by the lemma above, which means that if $0<\delta<2 \epsilon$, then there exists an integer $M$, such that for all $n>M$ we have $\left|G^{\prime}(n)+2 \epsilon\right|<\delta$, that is

$$
-2 \epsilon-\delta<G^{\prime}(n)<-2 \epsilon+\delta
$$

for all $n>M$. Let $a$ and $b$ be two integers such that $M<a<b$. Then

$$
G(b)-G(a)=\int_{a}^{b} G^{\prime}(n) d n<(b-a)(-2 \epsilon+\delta)<0 .
$$

If $a$ is fixed, it follows that $G(b)<G(a)+(b-a)(-2 \epsilon+\delta)<0$ for large $b$. Therefore there exists a positive integer $N>M$, such that for all $n>N$, we have $G(n)=F(\alpha, n)<0$.

We may assume that $N>688383$ so that from Lemma 2.2, part 3 we have $F(s, n)>0$. Moreover, from the same lemma we have $F(x, n)$ is decreasing for $x \geq 2 n$. As $s$ and $\alpha$ are both bigger than $2 n$, we have $s<\alpha$ for $n>N$ and the result follows.

Proof of Corollary 1.1. Let $\epsilon=\epsilon_{1}+\epsilon_{2}=0.5$. We will first show that for $n>688383$ we have $G^{\prime}(n)<0$.

Let $\epsilon_{1}=0.1$. It is easy to verify that for $n>688383$ we have

$$
\frac{1+\log n}{\log n\left(\log n+\log _{2} n-\log 2-\epsilon\right)}<\frac{\epsilon_{1}}{\log 2+\epsilon} .
$$

It follows that for all $n>688383$

$$
\begin{aligned}
\frac{n g(n)^{\prime}}{g(n)^{2}} & =\frac{(\log 2+\epsilon)(1+\log n)}{\log n\left(\log n+\log _{2} n-\log 2-\epsilon\right)^{2}} \\
& <\frac{\epsilon_{1}}{\log n+\log _{2} n-\log 2-\epsilon} .
\end{aligned}
$$

Next, we will show that $A+f^{\prime}(\alpha-n)-\log 2<\epsilon_{2}$.

Using Lemma 2.3, part 4 and Lemma 2.2 part 2 , we have

$$
\begin{aligned}
A+ & f^{\prime}(\alpha-n)-\log 2 \\
& <\log \left(\frac{\log (2.4 n)}{\log n}\right)+\frac{\log _{2}(2.4 n)}{\log (2 n)}+\frac{1.1}{\log n}+\frac{\log _{2}(1.4 n)}{\log ^{2} n} .
\end{aligned}
$$

Observe that for $n>36734$

$$
\log \left(\frac{\log (2.4 n)}{\log n}\right)<\frac{\epsilon_{2}}{5}
$$


as $\log \left(\frac{\log (2.4 n)}{\log n}\right)<\frac{\epsilon_{2}}{5}$ holds if $\frac{\log (2.4 n)}{\log n}<e^{\frac{\epsilon_{2}}{5}}$, that is if $2.4 n<n^{e^{\frac{\epsilon_{2}}{5}}}$. The above holds if $2.4<n^{e^{\frac{\epsilon_{2}}{5}-1}}$ or $n>36734$.

Computation yields that for $n>688383$

$$
\frac{\log _{2}(2.4 n)}{\log (2 n)}+\frac{1.1}{\log n}+\frac{\log _{2}(1.4 n)}{\log ^{2} n}<\frac{4 \epsilon_{2}}{5} .
$$

From equations (3.2)-(3.4) we have $A+f^{\prime}(\alpha-n)-\log 2<\epsilon_{2}$. From Lemma 2.3 part $3, L^{\prime}(\alpha-n)=U^{\prime}(\alpha-n)-f^{\prime}(\alpha-n)>\log (\alpha-n)+\log _{2}(\alpha-n)>$ $\log n+\log _{2} n$ and hence for $n>688383$ we have

$$
\frac{A+f^{\prime}(\alpha-n)}{-A+U^{\prime}(\alpha-n)-2 f^{\prime}(\alpha-n)}<\frac{\log 2+\epsilon_{2}}{\log n+\log _{2} n-\log 2-\epsilon_{2}} .
$$

As $\epsilon_{1}+\epsilon_{2}=\epsilon$, equations (3.1) and (3.5) give

$$
\begin{gathered}
\frac{A+f^{\prime}(\alpha-n)}{-A+U^{\prime}(\alpha-n)-2 f^{\prime}(\alpha-n)}+\frac{n g(n)^{\prime}}{g(n)^{2}} \\
<\frac{\log 2+\epsilon_{1}+\epsilon_{2}}{\log n+\log _{2} n-\log 2-\epsilon}=\frac{1}{g(n)} .
\end{gathered}
$$

From Lemma 2.3, part 2, noting that $\left(\frac{n}{g(n)}\right)^{\prime}=\frac{1}{g(n)}-\frac{n g(n)^{\prime}}{g(n)^{2}}$, we have $G^{\prime}(n)<0$ for all $n>688383$. Also, $G(688383)<0$ and hence we conclude that $G(n)<0$ for $n>688383$.

From Lemma 2.2, part 3 we have $F(s, n)>0$ and $F(x, n)$ is decreasing for $x \geq 2 n$. As $s$ and $\alpha$ are both bigger than $2 n$, it follows that $s<\alpha$ for $n>688383$. That the result holds for $43<n \leq 688383$ is a simple calculation.

REMARK 3.2. Similar results for lower bounds for $R_{n}$ can be given using $G(x, n)=L(x)-2 U(x-n+1)$ instead of $F(x, n)$.

\section{REFERENCES}

[1] C. Axler, Über die Primzahl-Zählfunktion, die n-te Primzahl und verallgemeinerte Ramanujan Primzahlen, Ph.D. thesis, 2013 (in German), http://docserv. uni-duesseldorf. de/servlets/DocumentServlet?id=26247

[2] C. Axler, On generalized Ramanujan primes, Ramanujan J. 39 (2016), 1-30.

[3] P. Dusart, Explicit estimates of some functions over primes, Ramanujan J. 45 (2018), $227-251$.

[4] S. Laishram, On a conjecture on Ramanujan primes, Int. J. Number Theory 6 (2010), 1869-1873.

[5] J. B. Paksoy, Derived Ramanujan primes $R_{n}^{\prime}$, http:/arxiv.org/abs/1210.6991.

[6] V. Shevelev, Ramanujan and Labos primes, their generalizations and classifications of primes, J. Integer Seq. 15 (2012), Article 12.1.1.

[7] J. Sondow, Ramanujan primes and Bertrand's postulate, Amer. Math. Monthly 116 (2009), 630-635.

[8] J. Sondow, J. W. Nicholson, T. D. Noe, Ramanujan primes: bounds, runs, twins, and gaps, J. Integer Seq. 14 (2011), Article 11.6.2. 
[9] A. Srinivasan, An upper bound for Ramanujan primes, Integers 14 (2014), paper A19.

[10] A. Srinivasan and J. Nicholson, An improved upper bound for Ramanujan primes, Integers 15 (2015), paper A52.

[11] S. Yang and A. Togbé, On the estimates of the upper and lower bounds of Ramanujan primes, Ramanujan J. 40 (2016), 245-255.

A. Srinivasan

Saint Louis University - Madrid Campus

Avenida del Valle 34, 28003 Madrid

Spain

E-mail: rsrinivasan.anitha@mgmail.com

P. Ares-Gastesi

Department of Applied Mathematics and Statistics

School of Business and Economics

Universidad CEU San Pablo

Madrid

Spain

E-mail: pablo.aresgastesi@ceu.es

Received: 26.1.2017.

Revised: 18.9.2017. 